\title{
Some Aspects of Profiling Electron Fields for Irradiation by Scattering on Foils
}

\begin{abstract}
Č. Šimáně, M. Vognar, D. Chvátil
With the use of formulae derived by Moliere and others the angular distributions of electrons scattered on Al foils have been calculated for electron energies $E_{0}=10,15,20,22$ and $25 \mathrm{MeV}$. Theoretical results are compared with flux density measurements of $22 \mathrm{MeV}$ electrons, scattered by a $0.16 \mathrm{~mm}$ Al foil, with a Faraday cup at distances 100 to $150 \mathrm{~cm}$ from the beam exit window, and at distances 110 and $130 \mathrm{~cm}$ for electrons scattered by Al foils 0.16, 0.26, 0.36, 0.46 and $0.66 \mathrm{~mm}$ in thickness. A strong dependence on the beam aperture, due to the broad beam scattering effects in air, was observed. Semiempirical formulae for beam apertures 1.56 and 4.6 degrees were derived, which reproduce well the experimental results of forward peak electron flux density (electrons $\cdot \mathrm{cm}^{-2} \cdot \mathrm{s}^{-1} \cdot \mu A^{-1}$ ) for $1 \mu \mathrm{A}$ of total beam current as functions of the distance from the beam exit window and of the thickness of the Al scattering foils.
\end{abstract}

Keywords: microtron, electron fields, electron scattering, scattering foils.

\section{Introduction}

High energy electron beams from linear accelerators or microtrons are strongly peaked forward. To get uniform irradiation fields for medical treatment or for other purposes, several techniques are used, either to destroy the forward peaking by scattering on foils [1] or to distribute the electron current by some scanning method.

There is a principal difference between these two ways of uniforming the electron flux over a given area. When the beam is scanned, only a relatively small part of the total beam current is lost at the boundaries of the field, inevitable in order to keep the mean current density within the boundaries in the prescribed limits. This is far from the case if scattering by foils is used for this purpose.

The results of the following considerations will to some extent have a qualitative character, as only a small angle scattering model is used for calculating the angular distribution of the electron beam scattered by foils, not including the radiation processes.

Let us suppose, as usual, a Gaussian angular distribution of the beam after scattering. Because the electron is not lost during the scattering process, the probability of finding the scattered electron in the total solid angle is equal to one.

The electron flux $F(\Theta)$ inside a cone of angular aperture $2 \Theta$, normalized to unity for $\Theta$ from 0 to $\pi$, can be written in the form

$$
F(\Theta)=\int_{0}^{\Theta} W(\Theta) \sin \Theta \mathrm{d} \Theta
$$

where $W(\Theta) \mathrm{d} \Theta$, the probability of an electron being scattered under an angle $\Theta$ into an interval $d \Theta$, is, according to the Moliere theory [2], given by an expression (with only the first term taken into account)

$$
W(\Theta) \mathrm{d} \Theta=\frac{2}{\Theta_{1}^{2} B} \exp \left(-\frac{\Theta^{2}}{\Theta_{1}^{2} B}\right) \mathrm{d} \Theta
$$

The constant factor before the exponential on the right side is the normalization factor and at the same time the amplitude of the Gaussian distribution.
The peak value of the distribution at $\Theta=0$ is inversely proportional to the width in radians of the angular distribution at $\mathrm{e}^{-1}$ height of the Gaussian. Therefore, knowing the amplitude, also the half-width can be deduced from it.

The constants $\Theta_{1}^{2}$ and $B$ depend on the thickness $x$, atomic number $Z$, atomic weight $A$ and density $s$ of the material of the scattering foil and on the energy $E_{0}$ of the primary beam (see Addendum).

Differential electron densities $W(\Theta)_{x}$ (Fig. 1) were calculated by formulae taken from [2], [3] and [4] for $\mathrm{Al}$ foils of thickness $x$ from 0.1 to $1 \mathrm{~mm}$ in $0.1 \mathrm{~mm}$ steps for energies $E_{0}=10,15,20$ and $25 \mathrm{MeV}$. It is interesting to note that the dependence of the peak value of the Gaussian distribution $A_{M}=2 /\left(\Theta_{1}^{2} B\right)$, resulting from the Moliere formulae, can with a high degree of precision be approximated by a simple function, which in the case of $\mathrm{Al}$ foils has the form

$$
\mathrm{A}_{M}=a \cdot x^{b}
$$

where $x$ is the $\mathrm{Al}$ thickness in $\mathrm{mm}$ of the scattering foil and the constants are $a=5002$ and $b=-1.162$.

\section{Experimental part}

For electron flux measurements in our experiments, the Faraday cup described in [5], was used. It was constructed for electron energies up to $25 \mathrm{MeV}$ and currents from $10^{-10}$ to $10^{-5} \mathrm{~A}$, with a circular, $0.1 \mathrm{~mm} \mathrm{Al}$ entry window of $\Phi=1.8 \mathrm{~cm}$ (window area $=2.54 \mathrm{~cm}^{2}$ ). The angular aperture of the input window of the Faraday cup as a function of the distance from the beam outlet window is represented in Fig. 2. If situated close to the beam outlet window of the microtron electron guideline, the Faraday cup receives the total electron beam, thus giving the value of the total electron flux. This value was used for calibration of the induction type current monitor, situated at the vacuum side before the beam exit window in the electron guideline, which served as the beam intensity monitor during the experiments.

The forward peak electron flux density $f_{\Theta=0}(L, x, \Gamma)$ $\left[\mathrm{n}_{\mathrm{e}} \mathrm{cm}^{-2} \mathrm{~s}^{-1} \mu \mathrm{A}^{-1}\right]$ per $1 \mu \mathrm{A}$ of electron beam current $\left(\mathrm{n}_{\mathrm{e}}\right.$ is the number of electrons, $x$ the scattering $\mathrm{Al}$ foil thickness in $\mathrm{mm}$ 

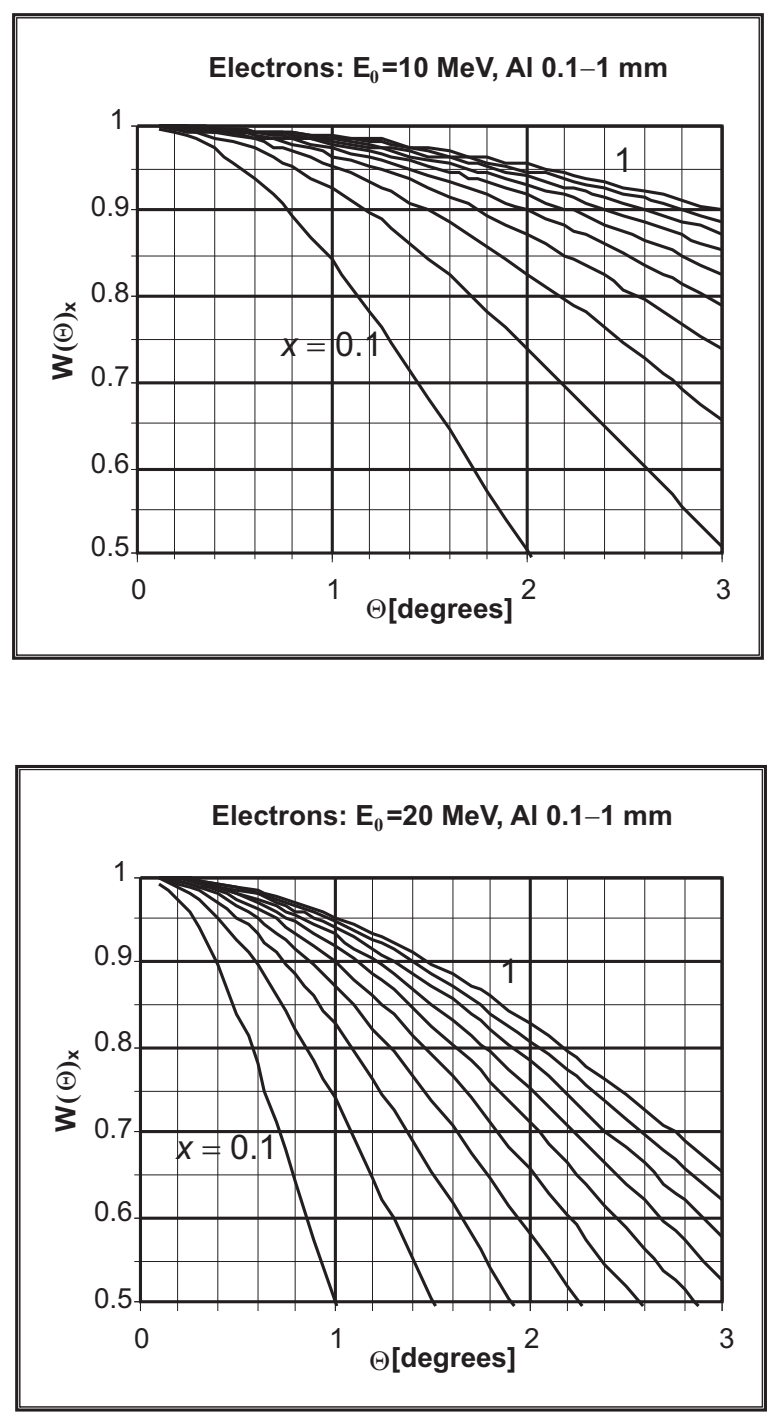
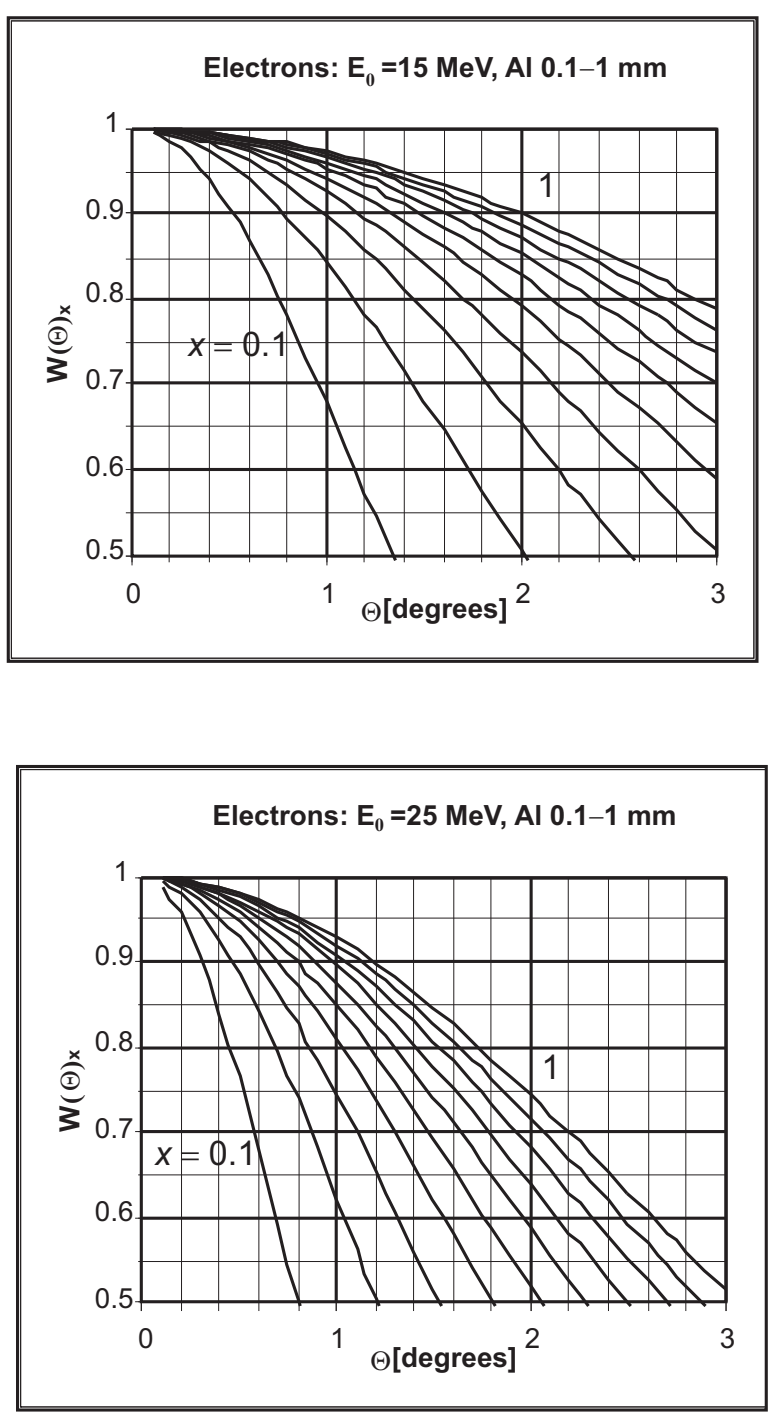

Fig. 1: Gaussian distributions $W(\Theta)_{x}$ of electrons scattered on Al foils of thickness $x$ (amplitude normalized to 1) for energies 10, 15, 20 and $25 \mathrm{MeV}$

and $\Gamma$ the beam aperture in degrees) was measured for $L$ (the distance from the beam exit window) from 110 to $150 \mathrm{~cm}$

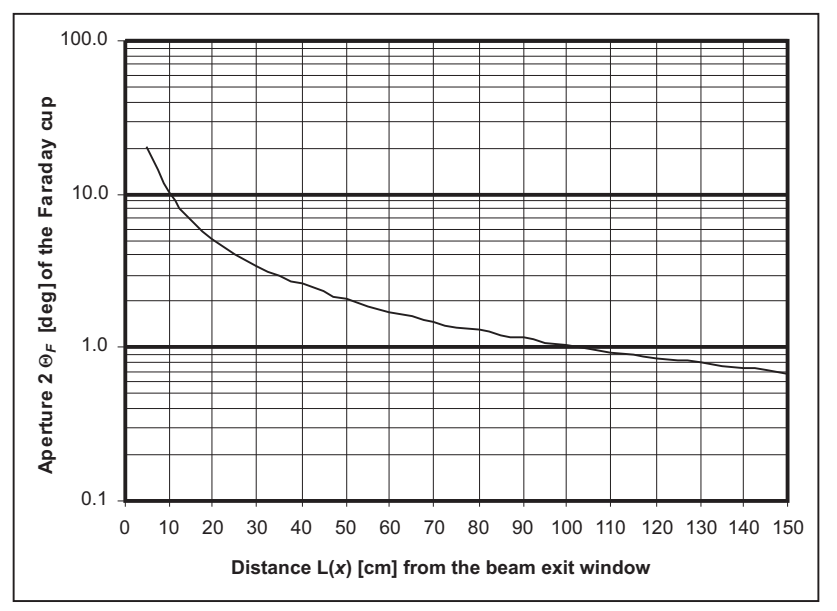

Fig. 2: Angular aperture $2 \Theta$ of the input window of the Faraday cup as a function of its distance from the beam outlet window in $10 \mathrm{~cm}$ steps, for $x=0.16 \mathrm{~mm}, \Gamma_{1}=1.57$ and $\Gamma_{2}=4.6$ degrees. To limit the beam aperture $\Gamma_{1}$ to 1.57 degree, a diaphragm of $\Phi=1.2 \mathrm{~cm}$ was placed at a distance $53 \mathrm{~cm}$ from the beam exit window. For $\Gamma_{2}=4.6$ degree a diaphragm of $\Phi=4.0 \mathrm{~cm}$ was situated at a distance of $60 \mathrm{~cm}$. The experimental results are presented as crosses in the graph in Fig. 4.

In another series of experiments, the electron flux densities were measured for two distances, $L=110$ and $130 \mathrm{~cm}$, with scattering foils $0.16,0.26,0.36,0.46$ and $0.66 \mathrm{~mm}$ for beam apertures $\Gamma=1.57$ and 4.6 degrees. The emplacement of the diaphragms and their diameters were the same as in the previous case. The results are presented as crosses in the graphs in Fig. 5 and Fig. 6.

The forward peak electron flux density $f_{\Theta=0}$ was calculated by dividing the current of the Faraday cup by the total beam current, by the area of its entry window equal to $2.54 \mathrm{~cm}^{2}$ and by the electron charge $1.6 \cdot 10^{-19} \mathrm{C}$. Finally, the electron flux density was reduced by a factor of $10^{-6}$ to obtain the results per $1 \mu \mathrm{A}$ of the total beam current. The mean square error of the mean value of five current readings in each measurement was less than $\pm 3 \%$. 


\section{Theoretical part}

We shall suppose that the electron beam before scattering is strictly parallel and has negligible radial dimensions. Then, for electron energy $E_{0}=22 \mathrm{MeV}$, used in our experiments, angular apertures corresponding to the heights $h=0.95,0.5$ and 1/e of the peak value of the Gaussian distribution of the scattered beams (Fig. 3) close behind the Al scattering foils can be calculated as a function of their thickness $x[\mathrm{~mm}]$. For the shortest distance in our experiments $L=110 \mathrm{~cm}$ of the Faraday cup from the beam outlet window, the angular aperture of the Faraday cup entry window is about $0.9^{\circ}$. Fig. 3 and Fig. 1 show that the inhomogeneity of the electron flux density at the Faraday cup entry opening is less than $\pm 5 \%$ in the case of $x>0,16 \mathrm{~mm}$ and less than $\pm 2.5 \%$ in the case of $x>0.26 \mathrm{~mm}$. In reality, the uniformity will be better, due to the scattering of electrons during their flight in air.

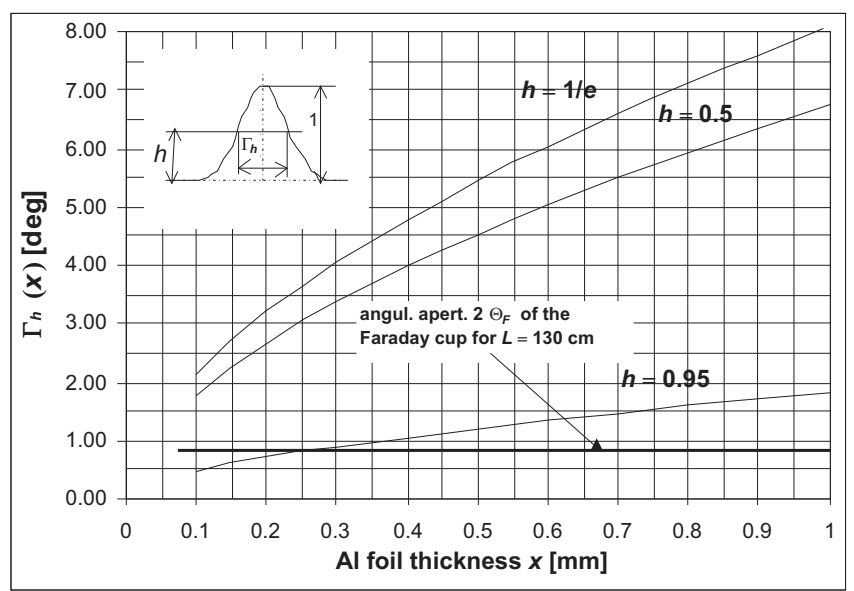

Fig. 3: Angular apertures $\Theta(x)_{h}$ of the beam width at the heighs $h=0.95,0.5$ and $1 / e$ of the amplitude of the Gaussian distribution of electrons scattered on $\mathrm{Al}$ foils of thickness $x$

Because the thickness of the air layer, when expressed in $\mathrm{g} / \mathrm{cm}^{2}$, in the electron flight path is comparable with the thickness of the scattering foil, it must be taken into account and a formula for calculating the electron flux density must contain a member that accounts for the scattering on air molecules. Unlike the scattering in thin Al foil, where the radial displacement of the electron in its volume is negligible, the calculation of the scattering in the air volume, if axial symmetric, is a three dimensional problem and radial displacements of the electrons cannot be neglected. The scattering must be considered as broad beam scattering. We tried to simplify the problem by assuming, as is usually done in similar problems e.g., in neutron or gamma scattering, that the build up effects can be accounted for an exponential decrease of the flux density with proper choice of the linear attenuation coefficient $\Sigma$ and by modification of the point source magnitude. The forward peak $(\Theta=0)$ electron flux density will then be of the form

$$
\begin{gathered}
f(L, x, \Gamma)_{\Theta=0}=A_{i}(x, \Gamma) \exp \left(-\Sigma_{i}(\Gamma) L\right) / L^{2}, \\
{\left[\mathrm{n}_{\mathrm{e}} \cdot \mathrm{cm}^{-2} \cdot \mathrm{s}^{-1} \mu \mathrm{A}^{-1}\right]}
\end{gathered}
$$

where $A$ depends on the foil thickness $x$ and the beam aperture $\Gamma$, and $\Sigma$ only on the beam aperture. The index $i$ denotes the beam aperture.

Using expressions (1) and (4), the $22 \mathrm{MeV}$ electron flux density at the entry of the Faraday cup has been calculated in dependence on distance $L$ in the range of 110 to $150 \mathrm{~cm}$.
With $\mathrm{A}_{1}=2.58 \cdot 10^{15}$ and $\mathrm{A}_{2}=2.28 \cdot 10^{15}\left[\mathrm{n}_{\mathrm{e}} \mathrm{s}^{-1} \mu \mathrm{A}^{-1}\right]$ in (4), which are very close to the peak value as calculated from the Moliere formula, and with $\Sigma_{1}$ and $\Sigma_{2}$ put equal to $0.00639 \mathrm{~cm}^{-1}$ resp. $0.0019 \mathrm{~cm}^{-1}$ for $\Gamma_{1}$ and $\Gamma_{2}$, formula (4) reproduces very well the experimental results for $x=0.16 \mathrm{~mm}$, as can be seen from Fig. 4 (full lines).

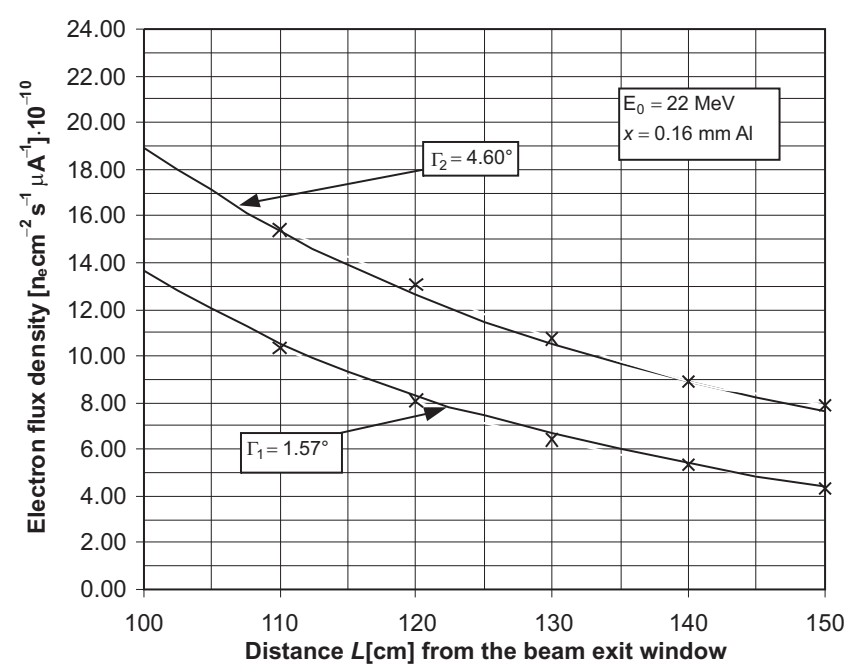

Fig. 4: Theoretical (full line) and experimental values (crosses) of forward peak flux density $f(L, x)_{\Theta=0}$ of electrons $E_{0}=22 \mathrm{MeV}$ scattered on $\mathrm{Al}$ foil $0.16 \mathrm{~mm}$ thick as functions of the distance from the beam exit window for two values of beam aperture $\Gamma_{1}=1.57$ and $\Gamma_{2}=4.60$ degree

Keeping $\Sigma_{1}$ and $\Sigma_{2}$ unchanged, the values of functions $A(x, \Gamma)$ were fitted on experimental results for $x=0.16,0.26$, $0.36,0.46$ and $0.56 \mathrm{~mm}$. We found that the set of values of $A$ selected in this way can be approximated by the functions $A(x)=5.46 \cdot 10^{14} x^{-0.8480}$ for beam aperture $\Gamma_{1}=1.57^{\circ}$ $A(x)=6.92 \cdot 10^{14} x^{-0.6525}$ for beam aperture $\Gamma_{2}=4.60^{\circ}$

Final expressions used for calculating the theoretical curves represented in the graphs in Fig. 5 and Fig. 6 are then

$$
\begin{aligned}
f(L, x)_{\Theta=0}= & 5.46 \cdot 10^{14} x^{-0.8480} \exp (-0.00639 L) / L^{2} \\
& \text { for } \Gamma_{1}=1.57^{\circ}
\end{aligned}
$$

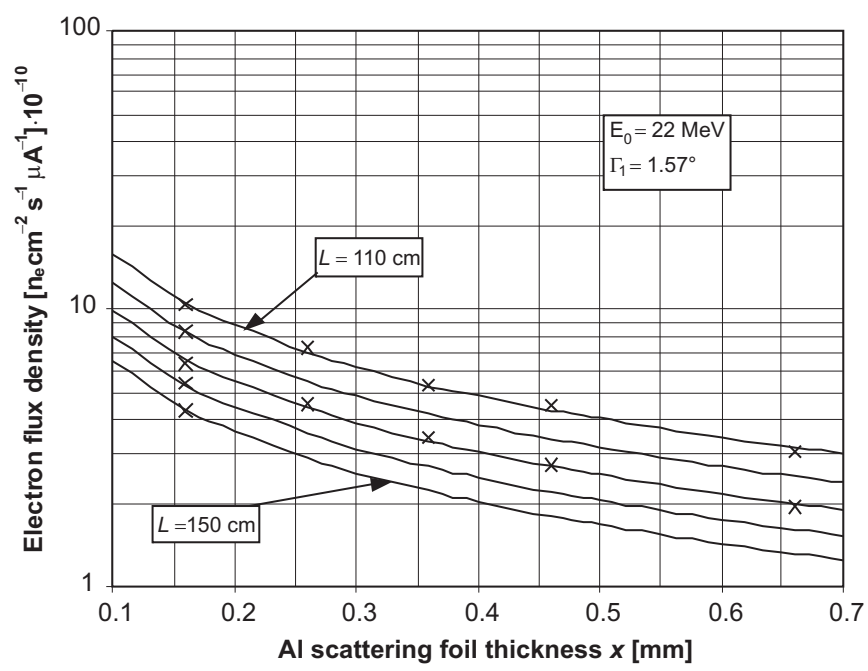

Fig. 5: Theoretical (full line) and experimental values (crosses) of forward peak flux density $f(L, x)_{\Theta=0}$ of electrons $E_{0}=22 \mathrm{MeV}$ as functions of the thickness of $\mathrm{Al}$ scattering foils for beam aperture $\Gamma_{1}=1.57$ degree at distances 110 to $150 \mathrm{~cm}$ from the beam exit window 


$$
\begin{aligned}
f(L, x)_{\Theta=0}= & 6.92 \cdot 10^{14} x^{-0.6525} \exp (-0.0019 L) / L^{2} \\
& \text { for } \Gamma_{2}=4.60^{\circ} .
\end{aligned}
$$

The deviations of the experimental points from the theoretical curves are in the order of several percent.

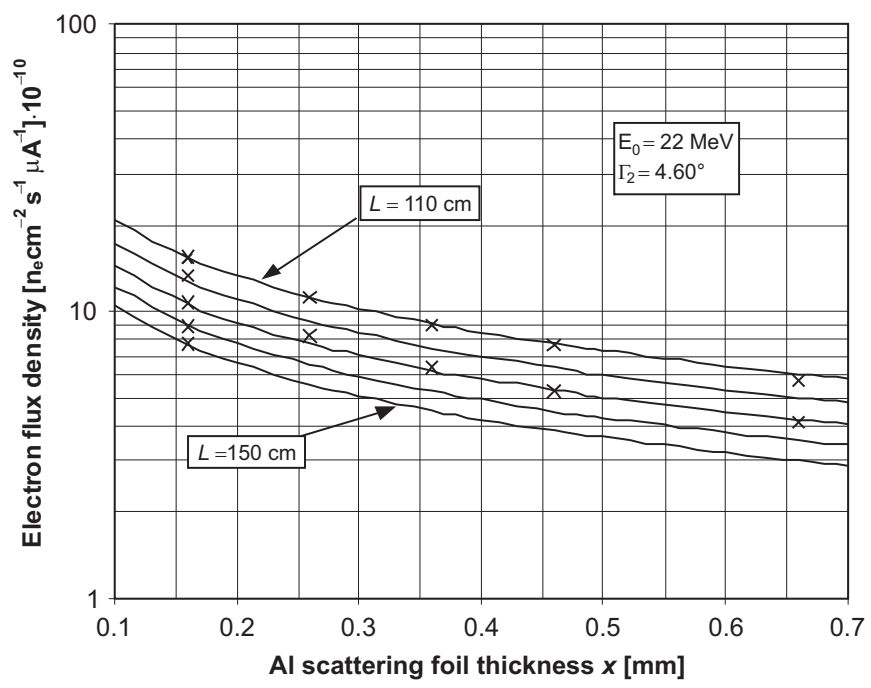

Fig. 6: Theoretical (full line) and experimental values (crosses) of electron peak flux density $f(L, x)_{\Theta=0}$ of electrons $E_{0}=22 \mathrm{MeV}$ as functions of the thickness of $\mathrm{Al}$ scattering foils for beam aperture $\Gamma_{1}=4.60$ degree at distances 110 to $150 \mathrm{~cm}$ from the beam exit window

The electron beam before scattering has a diameter of 3 to $5 \mathrm{~mm}$ and expressions (7) and (8) may be modified if other beam diameters are adjusted at the exit window. The ideal initial conditions supposed at the beginning of this part are not strictly fulfilled and in applying the formulae this must be respected.

\section{Conclusion}

The semiempirical expressions (7) and (8) were derived for two beam apertures from measurements in a rather limited range of thickness of $\mathrm{Al}$ scattering foils and of distances between the beam exit window and the Faraday cup. It is reasonable to suppose that similar simple functions can also be constructed from experimental measurements for other beam apertures. The strong dependence of the linear attenuation coefficients of air on beam apertures indicates a very important role of the geometrical configuration of diaphragms in the electron flight path. This role is not easily predictable from theory. It has also not been clarified how far the expressions can be extrapolated beyond the range of the variables for which they were derived. Similar expressions for other foil materials can probably be constructed from the same kind of measurements, perhaps excluding materials in which the probabilities of radiation processes and of small angle scattering are comparable. Further experiments with an extended range of variables $x, L$ and $\Gamma$ would be necessary to elucidate these questions.

The build-up effect in air evidently destroys the relation resulting from (2) between the half-width amplitude of the Gaussian distribution and its peak value. The distribution is no longer Gaussian, and intuitively should be broader. The build-up effect, if not limited by excessively small angular beam apertures, contributes not only to the electron flux density but also to the flattening of its distribution over the irradiation area.
At the beginning of our paper we stated that scanning of the beam would be preferable, if one intends to minimize electron beam losses while uniforming the field. This is true, provided that the electron flight path does not include air layers. Our experiments show that not only scattering in air may reduce the advantage of scanning over utilization of scattering foils, but also that scanning of the electron beam in air is always accompanied by broad beam build-up effects.

\section{Addendum}

This addendum provides a summary of expressions compiled from [2], [3] and [4], which served as a basis for the calculations of $B$ and $\Theta_{1}^{2}$. The physical units are the same as those used by the authors. The expressions are organized in the order that corresponds to the flow of the calculations.

$$
\begin{aligned}
& \alpha_{0}=Z e^{2} /(h c)=Z / 137 \\
& g(x)=E(x) / E_{0}=1-0.153(\ln x+19.45) \cdot s Z x /\left(A E_{0}\right) \\
& f(x)=\int_{0}^{x} g(x)^{-2} \mathrm{~d} x \\
& \xi^{2}=7800 \cdot Z^{1 / 3} \cdot(Z+1) \cdot f(x) /\left[A\left(1+3.35 \alpha_{0}^{2}\right)\right] \\
& B=\ln \left[1.1 \xi^{2} \ln \left(1.4 \xi^{2}\right)\right] \\
& K_{1}=8 \pi N_{0} Z(Z+1) e^{4} e=4.8 \cdot 10^{-10} \text { elst.j. } \\
& m c^{2}=8.0 \cdot 10^{-7} \mathrm{erg} \quad \mu=0.501 \mathrm{MeV} \\
& \varepsilon_{0}=E_{0} / \mu \quad \mu \quad{ }^{2} \\
& \Theta_{1}^{2}=K_{1} \cdot f(x) /\left(2 m c^{2} \varepsilon_{0}^{2}\right)
\end{aligned}
$$

$x=$ foil thickness $[\mathrm{cm}], Z=$ atomic number, $A=$ atomic mass, $s=$ density $\left[\mathrm{g} / \mathrm{cm}^{-3}\right], E=$ energy $[\mathrm{MeV}], E_{0}=$ initial electron energy $[\mathrm{MeV}], \varepsilon=$ energy in electron rest energy $\mu$ units, $m=$ electron mass [g], $e=$ charge of electron [elst.u.], $N_{0}=$ atoms $/ \mathrm{cm}^{3}$. For Al: $Z=13, A=26.98, s=2.699 \mathrm{~g} / \mathrm{cm}^{-3}$, $N_{0}=6.02 \cdot 10^{22} \mathrm{~cm}^{-3}$.

\section{References}

[1] Vognar, M., Šimáně, Č., Burian, A., Chvátil, D.: Electron and photon fields for dosimetric metrology generated by electron beams from a microtron. Nuclear Instruments and Methods in Physics Research A 380 (1996), pp. 613-617

[2] Moliere, G.: Z. Naturf. $3 a$ (1948), p. 78

[3] Kovalev, V. P.: Vtoritchnye izlutchenija uskoritelej elektronov. Atomizdat, Moskva 1979

[4] Kai Siegbahn: Beta and Gamma Ray Spectroscopy. North Holland Publishing Company, Amsterdam 1955

[5] Vognar, M., Šimáně, Č., Chvátil, D.: Faraday Cup for Electron Flux Measurements on the Microtron MT 25. Submitted for publication in Acta Polytechnica

Prof. Ing. Čestmír Šimáně, DrSc.

Ing. Miroslav Vognar

Ing. David Chvátil

Dept of Dosimetry \& Appl. of Ionizing Radiation

phone: +4202 2323657, +42022315212

fax: +42022320861

e-mail:Vognar@br.fjfi.cvut.cz

CTU, Faculty of Nuclear Science \& Physical Engineering Břehová 7, 11519 Praha 1

Czech Republic 